\title{
Laser Pulse Shortening via Zero-Dispersion Phase Matching of Parametric Raman Interactions in Crystals
}

\author{
Sergei N. Smetanin ${ }^{1, *}$, Michal Jelínek ${ }^{2}$ (D) Dmitry P. Tereshchenko ${ }^{1}$, Mikhail N. Ershkov ${ }^{3}$ and Václav Kubeček ${ }^{2}$ \\ 1 Research Center for Laser Materials and Technologies, Prokhorov General Physics Institute of the Russian \\ Academy of Sciences, Vavilova 38, 119991 Moscow, Russia; tereshenko.mitya2018@yandex.ru \\ 2 Department of Physical Electronics, Faculty of Nuclear Sciences and Physical Engineering, Czech Technical \\ University in Prague, Břehová 7, 11519 Prague, Czech Republic; michal.jelinek@fffi.cvut.cz (M.J.); \\ vaclav.kubecek@fjfi.cvut.cz (V.K.) \\ 3 Department of Laser Physics and Technologies, V. A. Degtyarev Kovrov State Technological Academy, \\ Mayakovskogo 19, 601910 Kovrov, Russia; ershkovm@yandex.ru \\ * Correspondence: ssmetanin@bk.ru; Tel.: + 7-499-5038793
}

check for updates

Citation: Smetanin, S.N.; Jelínek, M.; Tereshchenko, D.P.; Ershkov, M.N.; Kubeček, V. Laser Pulse Shortening via Zero-Dispersion Phase Matching of Parametric Raman Interactions in Crystals. Crystals 2021, 11, 19. https://doi.org/10.3390/ cryst11010019

Received: 8 December 2020 Accepted: 25 December 2020 Published: 29 December 2020

Publisher's Note: MDPI stays neutral with regard to jurisdictional claims in published maps and institutional affiliations.

Copyright: (C) 2020 by the authors. Licensee MDPI, Basel, Switzerland. This article is an open access article distributed under the terms and conditions of the Creative Commons Attribution (CC BY) license (https: / / creativecommons.org/ licenses/by/4.0/).

\begin{abstract}
We propose and study the conditions of zero-dispersion phase matching for parametric Raman interactions in birefringent crystals differing by anisotropy of zero-dispersion wavelength and allowing for the spectral tuning of the zero-dispersion phase-matching condition. We choose a highly birefringent crystal of calcite having a wide zero-dispersion anisotropy range for the demonstration of new effects of laser pulse shortening in parametric Raman lasers with spectrally tunable zerodispersion phase matching. We demonstrate the anti-Stokes $(1168 \mathrm{~nm})$ and multi-Stokes $(1629 \mathrm{~nm})$ picosecond pulse shortening and self-separation of single 80-ps ultra-short pulse from the zerodispersion phase-matched parametric Raman lasers that are based on the calcite crystal without using any electro-optical device.
\end{abstract}

Keywords: stimulated Raman scattering; Raman-active crystal; parametric Raman interaction; zerodispersion phase matching

\section{Introduction}

Crystals are the most common active media for nonlinear optics, because of not only their high nonlinearity and easy operation, but also the possibilities to maintain phase matching conditions of three- or four-photon nonlinear interactions due to the crystal birefringence (let us call it the birefringence phase matching) [1]. The only cubically nonlinear effects of two-photon stimulated inelastic scattering, including stimulated Raman scattering (SRS), do not require the maintenance of the phase matching condition for frequency conversion. However, even the SRS process, when it becomes multi-order, can be caused by four-photon parametric Raman interactions requiring the maintenance of the phase matching condition, which is limited by refractive index dispersion. Earlier, such axial parametric Raman generation of higher order SRS components has been obtained in gases having low dispersion [2]. Recently, such parametric Raman interactions in crystals allowed for the realization of strong shortening of generated radiation pulses under the conditions of the birefringence phase matching [3-6]. One more interesting possibility is using nanocrystals for which the phase matching condition is negligible for nonlinear optics [7-9].

Another great feature of solids is that the spectral dependence of the refractive index (as given by the Sellmeier equation) has the inflection point that corresponds to the zerodispersion wavelength [10]. The simplest approach for maintaining the four-photon-mixing phase-matching condition is to use the excitation wavelength that is close to the zerodispersion wavelength of the nonlinear solid-state medium. It allows for the realization of collinear phase matching (let us call it the zero-dispersion phase matching) for equally 
polarized waves without the walk-off problem in contrast to the birefringence phase matching in crystals. The zero-dispersion phase matching is known from nonlinear optics of optical fibers [11]. For example, a 1.319- $\mu \mathrm{m}$ Nd:YAG laser was used for phase-matched fourphoton mixing in a quartz glass fiber having a zero-dispersion wavelength of approximately $1.3 \mu \mathrm{m}[12]$.

In the present work, we propose using the conditions of the zero-dispersion phase matching for parametric Raman interactions in birefringent crystals that differ by anisotropy of zero-dispersion wavelength and allowing for the spectral tuning of the zero-dispersion phase-matching condition. We demonstrate possibilities of nanosecond-to-picosecond light pulse shortening and single ultra-short light pulse generation at intracavity antiStokes and multi-Stokes parametric Raman interactions in calcite at the zero-dispersion phase matching.

\section{Method of Laser Pulse Shortening via Parametric Raman Conversion}

The method of pulse shortening via parametric Raman conversion was proposed and studied in $[3,4]$. The second Stokes pulse was generated in the birefringent $\mathrm{Nd}: \mathrm{SrMoO} \mathrm{Hos}_{4}$ laser crystal via phase-matched parametric self-Raman laser interaction in the short temporal region of overlap of the depleted pump laser pulse and the first Stokes pulse generated by SRS self-conversion in the same laser crystal. It was explained by the fulfillment of the birefringence phase matching condition for orthogonally polarized four-photon mixing of single photons of the pump and second Stokes components with two photons of the first Stokes component. The second Stokes wavelength was defined as $\lambda_{2}=\left(2 \lambda_{1}{ }^{-1}-\lambda_{0}{ }^{-1}\right)^{-1}$, where $\lambda_{0}$ is the pump wavelength, $\lambda_{1}=\left(\lambda_{0}{ }^{-1}-v_{R}\right)^{-1}$ is the first Stokes SRS wavelength, and $v_{R}$ is the Raman frequency (in $\mathrm{cm}^{-1}$ ) of the active crystal. It was also found that the strong pulse shortening effect only occurred if the pumping process was intracavity differing by strong and spatially uniform depletion of pumping. As a result, the shortened pulse duration was close to the stage of depletion of pumping $\Delta t \approx 4.4 / g_{R} I_{p} c$ [4], where $g_{R}$ is the Raman gain of the active crystal, $I_{p}$ is the intensity of intracavity pumping, and $c$ is the speed of light.

We can say that similar four-photon mixing can take place for arbitrary three neighboring SRS components, i.e., the parametrically generated wavelength can be defined as

$$
\lambda_{j \pm 1}=\left(2 \lambda_{j}^{-1}-\lambda_{j \mp 1}^{-1}\right)^{-1}
$$

where $j$ is the SRS component number $(j=0$ is the fundamental pump laser wave, $j>0$ are the Stokes components, and $j<0$ are the anti-Stokes components). This process of collinear interaction will occur at the maintenance of the generalized phase matching condition [13]

$$
2 k_{j}=k_{j-1}+k_{j+1}
$$

where $k_{j}=2 \pi n_{j} / \lambda_{j}$ is the wavenumber of the $j$ th SRS component and $n_{j}$ is the refractive index for the $j$ th SRS component. Note that the wavelengths that appear to the right of the equal sign in Equation (1) can be considered as the pump and Stokes components of the two-photon SRS process of $j$ th order that should be the intracavity SRS process to obtain strong pulse shortening at the parametrically generated wavelength $\lambda_{j \pm 1}$.

Figure 1 demonstrates the pulse shortening mechanism via parametric Raman conversion for the cases of Q-switched and mode-locked pump lasers.

Figure 1 shows that the anti-Stokes or second Stokes pulse is parametrically generated in the short temporal region, where all of the radiation components overlap, because it is the four-photon process. The names of the light components in Figure 1 can be considered from the point of view of the $j$ th order SRS process according to Equation (1), and the parametrically generated component can be a higher order SRS component from the fundamental pump laser wave $(j=0)$. In the case of the nanosecond pump pulse (Figure 1a) that isgenerated by a Q-switched laser, the parametrically generated pulse has a duration that is close to the pump pulse depletion stage having duration in a range of 
picoseconds. This mechanism that was proposed and initially studied in [3,4] has recently been confirmed by space-dependent mathematical modeling in [14].

(a) Q-switched laser
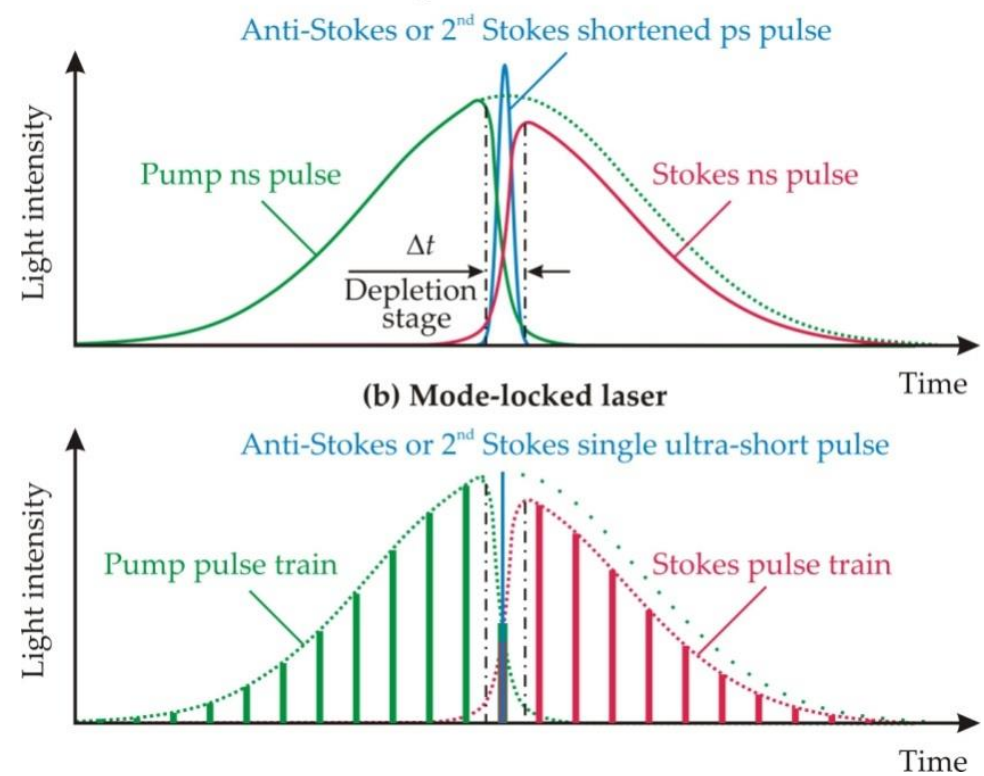

Figure 1. The pulse shortening mechanism via parametric Raman conversion for the cases of (a) Q-switched and (b) mode-locked pump lasers.

In the case of a Q-switched and mode-locked laser operation (Figure 1b), we can also obtain self-separation of a single ultra-short light pulse from the ultra-short pulse train of the mode-locked laser without any electro-optical device.

\section{Method of Tunable Zero-Dispersion Phase Matching for Parametric Raman Interaction in Crystals}

Refractive index dispersion is the main issue of fulfillment of the phase matching condition (2) and, therefore, we have $n_{j-1}>n_{j}>n_{j+1}$ (for normal dispersion), leading to the $j$ th order parametric Raman process wave mismatch [13]

$$
\Delta k_{j}=k_{j-1}+k_{j+1}-2 k_{j}=\left(n_{j-1}+n_{j+1}-2 n_{j}\right) 2 \pi \lambda_{j}^{-1}+\left(n_{j-1}-n_{j+1}\right) 2 \pi v_{R}
$$

It is necessary to note that the form of Equation (3) is close to the difference approximation of the second derivative of the wavenumber on the $j$ th radiation component frequency: $\Delta k_{j}=k\left(\omega_{j}+\omega_{R}\right)+k\left(\omega_{j}-\omega_{R}\right)-2 k\left(\omega_{j}\right) \approx \omega_{R}{ }^{2} \mathrm{~d}^{2} k / \mathrm{d} \omega_{j}{ }^{2}$, where $\omega_{j}=2 \pi c / \lambda_{j}$ and $\omega_{R}=2 \pi v_{R} c$ [15]. It means that $\Delta k_{j} \approx 0$, when $\mathrm{d}^{2} k / \mathrm{d} \omega_{j}^{2}=0$ corresponding to $\lambda_{j} \approx \lambda_{d}$, where $\lambda_{d}$ is the zero-dispersion wavelength. The remarkable property of such zero-dispersion phase matching is that it is noncritical to angular mismatch, as it was analyzed in [6].

Let us choose a typical crystalline material for our consideration. We have chosen calcite $\left(\mathrm{CaCO}_{3}\right)$-a well-known highly birefringent natural crystal having Raman activity with the Raman frequency of $v_{R}=1086 \mathrm{~cm}^{-1}$ and Raman gain $g_{R}$ of $2 \mathrm{~cm} / \mathrm{GW}$ and $0.3 \mathrm{~cm} / \mathrm{GW}$ at $1.06-\mu \mathrm{m}$ and $1.3-\mu \mathrm{m}$ pumping, respectively [5]. The first crystalline parametric Raman converter was based namely on this crystal [16], generating anti-Stokes cones at non-collinear phase matching, but it is not interesting for applications. The birefringence phase matching for axial parametric Raman interaction has also been maintained, at first, namely in calcite [17]. Recently, this crystal was used for high-beam-quality anti-Stokes parametric Raman generation in an optical cavity at the birefringence phase matching [5]. 
The Sellmeier equations for the dispersion curves of a negative uniaxial calcite crystal in the range of $\lambda=0.2-2.2 \mu \mathrm{m}$ are presented in [18]:

$$
\begin{gathered}
n_{\mathrm{o}}^{2}=1+\frac{0.8559 \lambda^{2}}{\lambda^{2}-0.0588^{2}}+\frac{0.8391 \lambda^{2}}{\lambda^{2}-0.141^{2}}+\frac{0.0009 \lambda^{2}}{\lambda^{2}-0.197^{2}}+\frac{0.6845 \lambda^{2}}{\lambda^{2}-7.005^{2}}, \\
n_{\mathrm{e}}^{2}=1+\frac{1.0856 \lambda^{2}}{\lambda^{2}-0.07897^{2}}+\frac{0.0988 \lambda^{2}}{\lambda^{2}-0.142^{2}}+\frac{0.317 \lambda^{2}}{\lambda^{2}-11.468^{2}} .
\end{gathered}
$$

Figure 2 shows the dependences of the ordinary wave refractive index $n_{\mathrm{o}}$ and the ordinary wave mismatch $\Delta k_{j}$ on the jth component wavelength $\lambda_{j}$ that was calculated from Equations (3) and (4) for calcite.

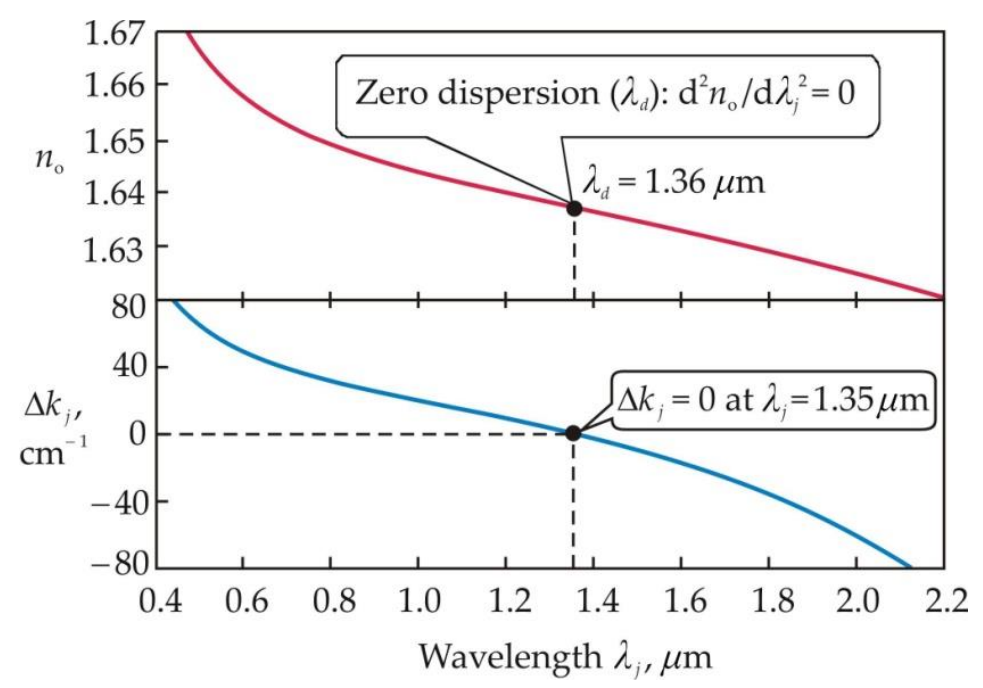

Figure 2. The calculated dependences of the ordinary wave refractive index $n_{\mathrm{o}}$ and the ordinary wave mismatch $\Delta k_{j}$ on the wavelength $\lambda_{j}$ for calcite.

It can be seen from Figure 2 that the ordinary wave mismatch $\Delta k_{j}$ is positive in the positive group velocity dispersion range $\left(\lambda_{j}<\lambda_{d}\right)$ and it is negative in the negative group velocity dispersion range $\left(\lambda_{j}>\lambda_{d}\right)$. The wave mismatch $\Delta k_{j}$ amounts to a value of zero at a wavelength $\lambda_{j}=1.35 \mu \mathrm{m}$, which is quite a bit less than the zero-dispersion wavelength $\lambda_{d}=1.36 \mu \mathrm{m}$.

It is necessary to note that, in highly birefringent crystals, we have not only anisotropy of refractive index, but also anisotropy of the zero-dispersion wavelength. Figure 3 demonstrates the possibility of tuning of the zero-dispersion wavelength for extraordinary waves via rotation of the calcite crystal while using the dependence of extraordinary wave refractive index on the propagation angle $\Theta[19]$ :

$$
n_{\mathrm{e}}(\Theta)=\frac{n_{\mathrm{e}} n_{\mathrm{o}}}{\sqrt{n_{\mathrm{o}}^{2} \sin ^{2} \Theta+n_{\mathrm{e}}^{2} \cos ^{2} \Theta}}
$$

where the principal values of refractive index $n_{\mathrm{e}}$ and $n_{\mathrm{o}}$ depend on the wavelength according to Equation (4). Therefore, while using rotation of the calcite crystal, we can tune the zero-dispersion wavelength from $\lambda_{d}=1.36 \mu \mathrm{m}$ at $\Theta=0(\mathbf{E} \perp c$, Figure 3a) up to $\lambda_{d}=1.39 \mu \mathrm{m}$ at $\Theta=28^{\circ}$, corresponding to the incidence angle of $49^{\circ}$ (Figure $3 \mathrm{~b}$ ), and further up to $\lambda_{d} \approx 1.76 \mu \mathrm{m}$ at $\Theta=90^{\circ}$ (E $\| c$, Figure 3c). We can summarize that we can tune $\lambda_{d}$ into $\lambda_{j}$ in order to obtain zero-dispersion phase matching of parametric Raman interaction for arbitrary $\lambda_{j}$ in the zero-dispersion anisotropy range of 1.36-1.76 $\mu \mathrm{m}$. 


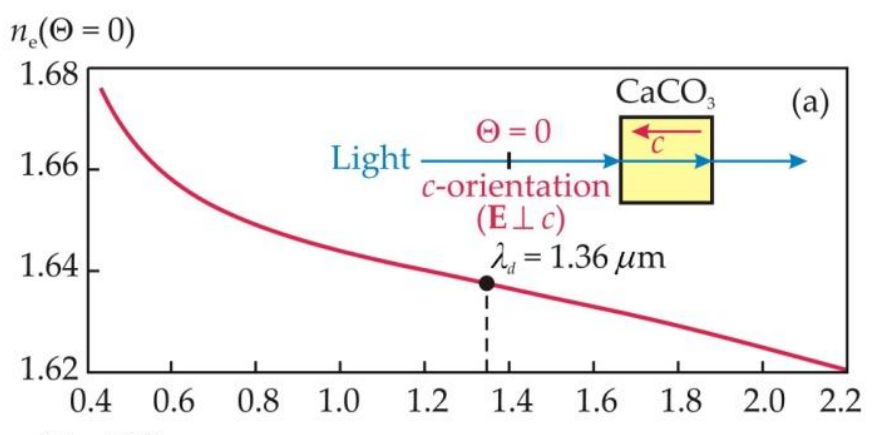

$n_{\mathrm{e}}\left(\Theta=28^{\circ}\right)$

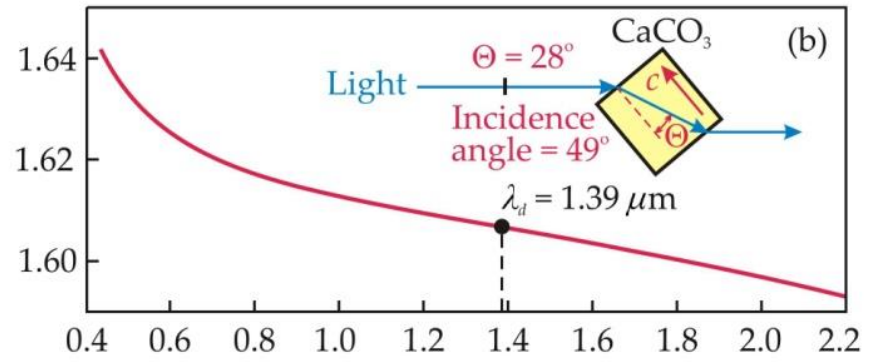

$n_{\mathrm{e}}\left(\Theta=90^{\circ}\right)$

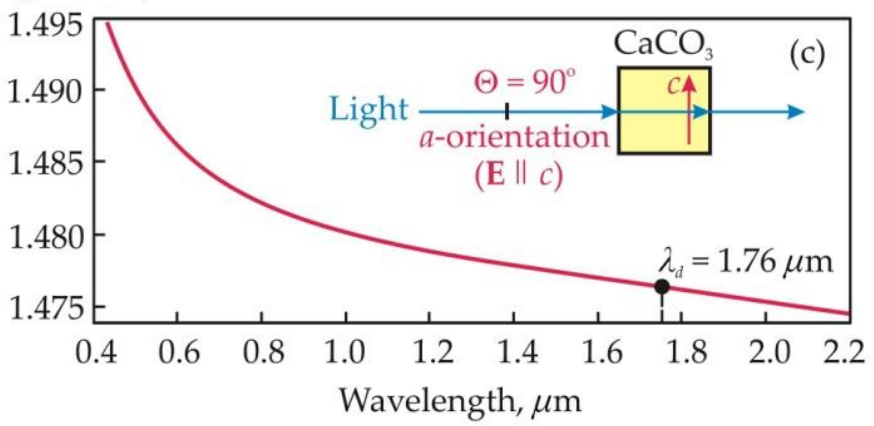

Figure 3. The calculated dependences of the extraordinary wave refractive index $n_{\mathrm{e}}(\Theta)$ on the wavelength for the calcite $\left(\mathrm{CaCO}_{3}\right)$ crystal at the propagation angles of (a) $\Theta=0,(\mathbf{b}) \Theta=28^{\circ}$, and $(\mathbf{c}) \Theta=90^{\circ}$.

We believe that the zero-dispersion anisotropy range should be wide, not only for calcite, but also for other highly birefringent crystals, for example, for positive uniaxial $\mathrm{YVO}_{4}$ and $\mathrm{GdVO}_{4}$ crystals having Raman activity [20] and also possibility of doping by laser ions for self-Raman operation [21].

\section{Intracavity Parametric Raman 1168-nm Anti-Stokes Laser with Ordinary Wave Zero-Dispersion Phase Matching in Calcite}

Firstly, we have experimentally studiedphase-matched parametric Raman anti-Stokes generation at the zero-dispersion wavelength of $\lambda_{d}=1360 \mathrm{~nm}$ for nonlinear interaction of ordinary waves in calcite (Figure 2). We used the calcite crystal (natural, VIS first grade, Siberian field, Russia) withdimensions of $10 \times 30 \times 33 \mathrm{~mm}^{3}$, where the optical axis (c) was oriented along the $33-\mathrm{mm}$ long side. The $10 \times 30 \mathrm{~mm}^{2}$ (c-cut) and $30 \times 33 \mathrm{~mm}^{2}$ (a-cut) faces were optically polished. In this experiment, we used excitation through the $30 \times 33 \mathrm{~mm}^{2}$ face of the crystal. The refractive index calculation from the presented Sellmeier equations was confirmed by the test of Brewster effect in our calcite sample. We chose a Nd:YAG laser at a wavelength of $\lambda_{0}=1338 \mathrm{~nm}$ as the pump laser, because its wavelength is close to the wavelength of $1350 \mathrm{~nm}$ (see Figure 2) of the zero-dispersion phase matching $\left(\Delta k_{0}=0\right)$ for the zero order parametric Raman process (see Equation (2) where $j=0$ ). Figure 4 shows the parametric Raman laser oscillator scheme, photo, and measured output radiation spectrum. The laser was at first demonstrated by us in [22], where it was studied at the strongest Q-switching regime. The laser consisted of the active Nd:YAG laser crystal, nonlinear 
calcite Raman crystal, and passive V:YAG Q-switch in the plan-concave two-mirror cavity. The laser oscillator active medium was formed by the Nd:YAG slab crystal of trapezoidal shape with $\mathrm{Nd}^{3+}$ concentration of $2.4 \mathrm{at} . \%$. Two $5 \times 2 \mathrm{~mm}^{2}$ end faces of the crystal were angled at 68 degrees. This configuration allowed for the laser cavity beam incident at the Brewster angle to these faces and experience total internal reflection from the laser-diode pumped face, resulting in the horizontally-polarized laser generation. The pumping face of $30 \times 2 \mathrm{~mm}^{2}$ was antireflection coated for the pumping wavelength of $808 \mathrm{~nm}$. As the pumping source, a linear single bar quasi-continuous laser diode array with the fast axis collimation and maximum output peak power of $130 \mathrm{~W}$ was utilized. The pumping pulse duration was varied in the range from 250 to $470 \mu$ s and the pulse repetition rate was $10 \mathrm{~Hz}$. In order to increase the laser medium gain, the pump beam spot size at the active crystal face was decreased in the vertical axis down to $\sim 200 \mu \mathrm{m}$ while using a cylindrical lens $(\mathrm{f}=5 \mathrm{~cm})$. In our initial experiment [22], the pumping beam spot was approximately $2 \mathrm{~cm}$ (in the horizontal axis) $\times 920 \mu \mathrm{m}$ (in the vertical axis). The antireflection-coated V:YAG crystals with the initial transmittance of 74 and $66 \%$ were used in order to achieve passively Q-switched regime. The 10-mm long $a$-cut calcite nonlinear crystal was placed inside the oscillator under the Brewster angle at vertical orientation of the crystal optical axis allowing for horizontally polarized ordinary wave mixing at $\mathrm{E} \perp c$.

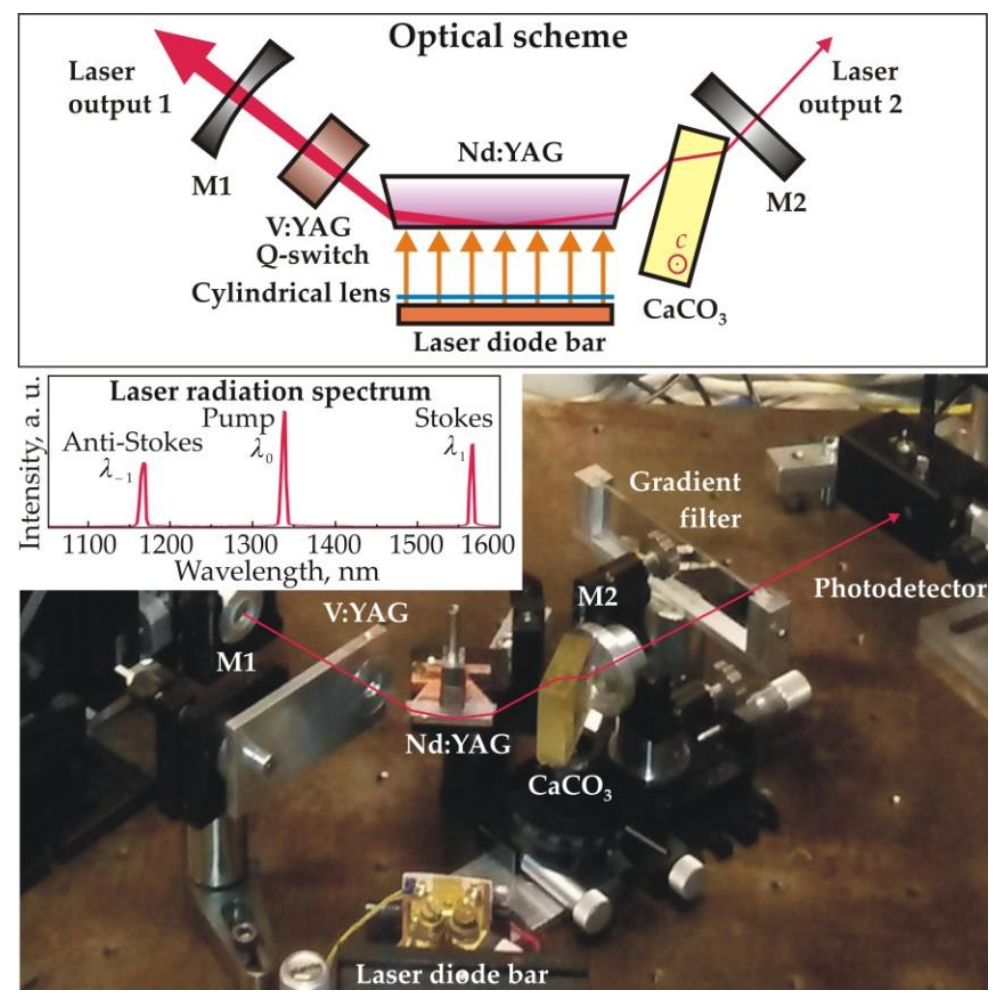

Figure 4. The intracavity parametric Raman anti-Stokes laser system scheme, photo, and measured radiation spectrum.

The laser cavity was formed by the concave mirror M1 (curvature radius of $150 \mathrm{~mm}$ ) and the flat mirror M2. Table 1 presents the cavity mirror reflectivities at the generation wavelengths. We can see that the cavity had high quality factor for fundamental lasing wavelength at $\lambda_{0}=1338 \mathrm{~nm}$ and intracavity SRS conversion into the 1st Stokes component at $\lambda_{1}=\left(\lambda_{0}{ }^{-1}-v_{R}\right)^{-1}=1565 \mathrm{~nm}$. The mirrors M1 and M2 had reflectivities at wavelengths of $82 \%$ and $30 \%$, respectively, in order to obtain nonlinear cavity dumping at the antiStokes wavelength of $\lambda_{-1}=\left(2 \lambda_{0}^{-1}-\lambda_{1}{ }^{-1}\right)^{-1}=\left(\lambda_{0}^{-1}+v_{R}\right)^{-1}=1168 \mathrm{~nm}$ (see Table 1). The mirrors also had high transmittance at the wavelengths of $1064 \mathrm{~nm}$ (unwanted $\mathrm{Nd}^{3+}$-laser transition) and $1886 \mathrm{~nm}$ (unwanted 2nd Stokes SRS component, $\lambda_{2}=\left(\lambda_{0}^{-1}-2 v_{R}\right)^{-1}$ ) in order to prevent their generation. 
Table 1. The anti-Stokes laser cavity mirror reflectivities at the radiation wavelengths.

\begin{tabular}{cccc}
\hline Mirror & $R @ \mathbf{1 1 6 8} \mathbf{n m}$ & $\boldsymbol{R} @ \mathbf{1 3 3 8} \mathbf{n m}$ & $\boldsymbol{R} @ \mathbf{1 5 6 5} \mathbf{n m}$ \\
\hline M1 & $82 \%$ & $99.8 \%$ & $94 \%$ \\
M2 & $30 \%$ & $99.8 \%$ & $96 \%$ \\
\hline
\end{tabular}

The mirror M1 was placed on a precise translation stage. It allowed for prolonging the cavity length up to the hemispherical cavity stability edge (the plan-concave cavity length was close to the M1 mirror curvature radius of $150 \mathrm{~mm}$ ) to obtain apassive self-mode locking regime. The nonlinear calcite crystal was placed in the intracavity beam waist near the flat mirror M2 where both the nonlinear effects of Kerr-lens mode-locking and parametric Raman interaction in the same calcite crystal were the most efficient. We realized not only self-mode-locked, but also passively Q-switched regime by the V:YAG saturable absorber, in order to increase the fundamental laser intracavity radiation intensity to reach the SRS threshold. Passive V:YAG Q-switching additionally helped us to obtain generation of the fundamental laser radiation only at the wavelength of $1338 \mathrm{~nm}$ close to the zero dispersion of the calcite crystal without lasing at the wavelength of $1319 \mathrm{~nm}$ (having the same emission cross-section in the Nd:YAG crystal [21]), because of the higher initial transmittance of the V:YAG crystal at the 1338-nm wavelength than at the 1319-nm wavelength.

We started our study from getting the stable self-mode-locking regime in the Nd:YAG/ calcite laser with the $T_{0}=74 \% \mathrm{~V}: Y A G \mathrm{Q}$-switch. The diode pumping pulse duration and peak power were $250 \mu$ s and $130 \mathrm{~W}$, respectively. Single Q-switched and mode-locked pulse train was generated at the end of the pumping pulse. Subsequently, we used optically denser V:YAG Q-switch with initial transmittance of $T_{0}=66 \%$. In order to obtain lasing, we had to prolong the diode pumping pulse duration up to $470 \mu$ s at the same peak power of $130 \mathrm{~W}$.

Figure 5 demonstrates oscillograms of the separated output radiation spectral components registered simultaneously while using two InGaAs PIN photodiodes EOT ET-3500 (Electro-Optics Technology, Inc., Traverse City, MI, USA) (analog bandwidth > $12.5 \mathrm{GHz}$, rise-time $<35$ ps) that were connected to the oscilloscope LeCroy-Teledyne SDA 9000 (Teledyne LeCroy, Inc., Milpitas, CA, USA) (analog bandwidth $9 \mathrm{GHz}$, sampling rate $40 \mathrm{GS} / \mathrm{s}$ ). We can see that the self-mode-locked pulse train containing all of the output radiation spectral components (see the red lines in Figure 5) had an additional peak at the envelope trailing edge explained by the SRS radiation generation. The SRS radiation included not only the Stokes component $\left(\lambda_{1}=1565 \mathrm{~nm}\right)$ generated by SRS, but also the parametrically generated anti-Stokes component $\left(\lambda_{-1}=1168 \mathrm{~nm}\right)$, which was also registered separately (see the blue lines in Figure 5; also see the measured output radiation spectrum in Figure 4). Because of mode selection in the optically dense Q-switch, we realized the locking of only several longitudinal modes of the laser cavity with relatively long duration of the fundamental laser pulses of about 400 ps (undepleted fundamental laser radiation in Figure 5), but intracavity SRS conversion took place with strong SRS pulse shortening.

In the case of the Q-switch with higher transmittance of $T_{0}=74 \%$, the SRS radiation pulse train contained ten pulses, as one can see from Figure $5 \mathrm{a}$ (see the red line in Figure 5a). However, the anti-Stokes pulse train contained just six pulses (see the blue line in Figure 5a), and so the anti-Stokes pulse train was shortened in comparison with the Stokes pulse train. It can be explained by the mechanism of ultra-short pulse self-separation that is presented in Figure 1b, but, in the experiment, the fundamental radiation depletion stage was long, resulting in the separation of not only one, but several ultra-short pulses of the anti-Stokes radiation. Additionally, note that the individual SRS pulses were strongly shortened in comparison with the fundamental laser pulses. The duration of Stokes pulses was measured to be $\sim 160$ ps (FWHM). The anti-Stokes pulses were even shorter of about 120 ps. 

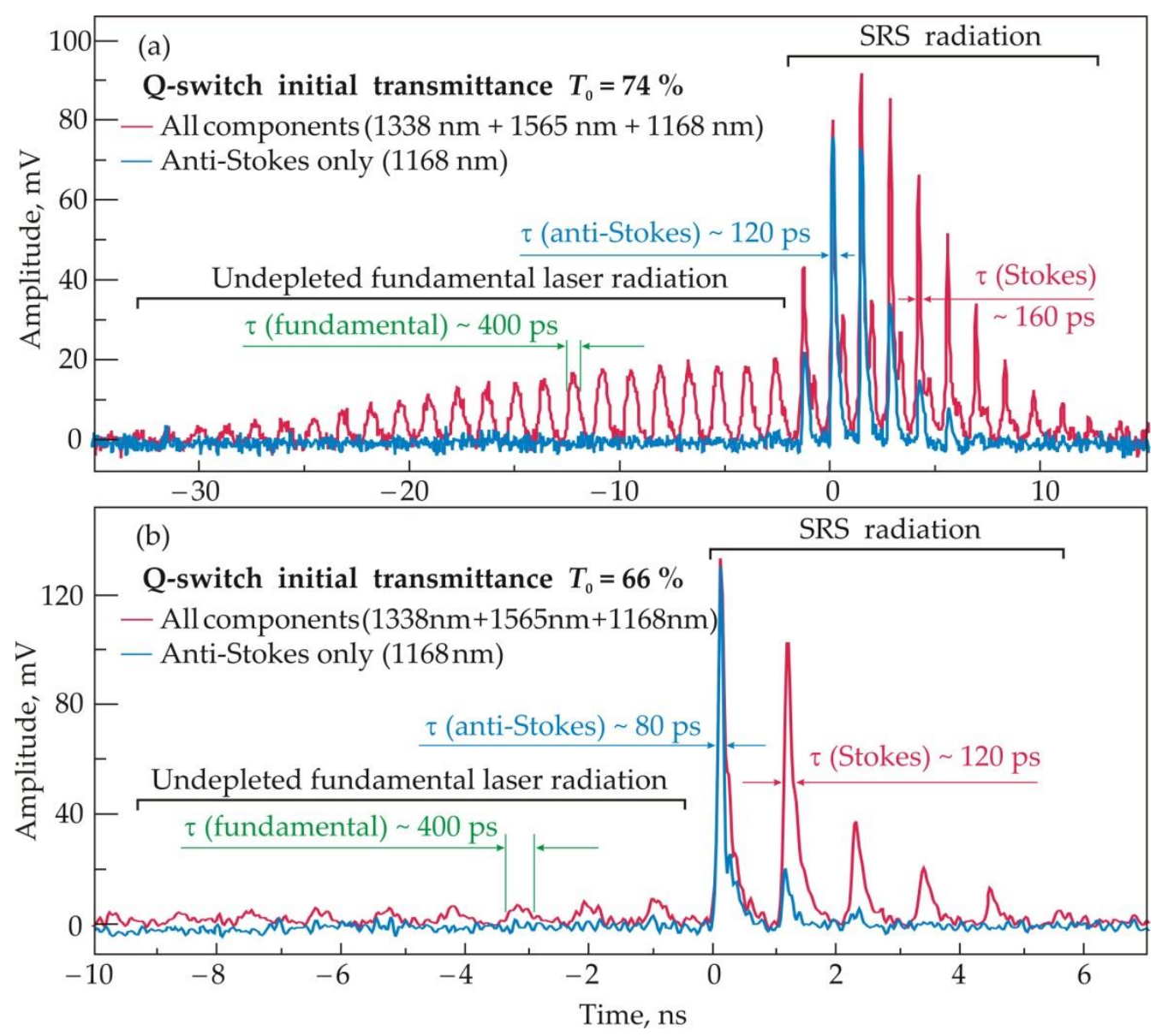

Figure 5. Oscillograms of the output radiation spectral components of the intracavity parametric Raman anti-Stokes laser with the (a) $74 \%$ and (b) $66 \%$ passive Q-switch.

It can be seen from Figure $5 \mathrm{~b}$ that, in the case of using the Q-switch with lower transmittance of $T_{0}=66 \%$, the Q-switching regime became very strong with the generation of significantly more intense SRS pulses in comparison with the undepleted fundamental laser pulses, and also in comparison with the case of the 74-\% Q-switch. The number of pulses in the SRS pulse train decreased down to five (the red line in Figure 5b). Additionally, we have really obtained self-separation of a single ultra-short anti-Stokes pulse (the blue line in Figure $5 \mathrm{~b}$ ) with the shortest pulse duration of $\sim 80$ ps or may be even shorter, because $\sim 80 \mathrm{ps}$ is a resolution limit of the oscilloscope-photodiode system. This optimized result is better than our initial result presented in [22], where we obtained self-separation of a few anti-Stokes pulses with longer pulse duration $(\sim 120 \mathrm{ps})$.

We directly measured the pulse train energies of the separated (fundamental, Stokes, anti-Stokes) radiation components behind both laser cavity mirrors. Energy was measured while using a highly sensitive probe Coherent J-10MB-LE (Coherent, Inc., Salem, NH, USA) (energy range $300 \mathrm{~nJ}-600 \mu \mathrm{J}$ ) connected to an oscilloscope.

Using the $T_{0}=74 \%$ V:YAG Q-switch with the diode pump pulse duration and peak power of $250 \mu$ s and $130 \mathrm{~W}$, respectively, we measured the overall $(1338 \mathrm{~nm}+1565 \mathrm{~nm}+1168 \mathrm{~nm})$ output energy that was summed from both of the cavity mirrors that amounted to $12.5 \mu \mathrm{J}$. The separated 1565-nm Stokes output energy was $3 \mu \mathrm{J}$. The 1168-nm anti-Stokes output energy was $0.5 \mu \mathrm{J}$. Accordingly, the Stokes and anti-Stokes output energies were $24 \%$ and $4 \%$, respectively, from the overall output radiation energy. Taking the Stokes and anti-Stokes pulse train envelopes into account, we also estimated the energy of the most intensive individual ultra-short pulses, which amounted 0.6 and $0.2 \mu \mathrm{J}$ for the Stokes and anti-Stokes components, respectively, i.e., the anti-Stokes ultra-short pulse energy was threetimes lower than the Stokes ultra-short pulse energy. 
Using the $T_{0}=66 \% \mathrm{~V}: Y A G$ Q-switch with $470 \mu \mathrm{s}, 130 \mathrm{~W}$ diode pumping, the overall output energy amounted to $16 \mu \mathrm{J}$. The 1565-nm Stokes output energy was $6 \mu \mathrm{J}$. The 1168-nm anti-Stokes radiation was generated as the single pulse with output energy of $1.8 \mu \mathrm{J}$. Thus, the Stokes and anti-Stokes output energies increased up to $38 \%$ and $11.3 \%$, respectively, from the overall output energy. Taking the Stokes pulse train envelope into account, we again estimated the energy of the most intensive individual Stokes pulse, which amounted to approximately $2.6 \mu \mathrm{J}$. Therefore, the anti-Stokes pulse energy became 1.4 times lower than the most intense Stokes pulse energy, but the peak power of the anti-Stokes pulse exceeded the Stokes pulse peak power, because the anti-Stokes pulse duration was more than 1.4 times shorter $(<80 \mathrm{ps})$.

\section{Extracavity Parametric Raman 1629-nm Third Stokes Laser with Extraordinary Wave Zero-Dispersion Phase Matching in Calcite}

As the second experiment, we experimentally investigated the phase-matched parametric Raman multi-Stokes generation at tuning of the zero-dispersion wavelength rotating the calcite crystal (Figure 3). We used the same calcite sample with excitation through its $10 \times 30 \mathrm{~mm}^{2}$ face at a controllable angle of incidence.

This parametric Raman laser was pumped by a laboratory-designed oscillator-amplifier $\mathrm{Nd}$ :YAG laser system generating at $\lambda_{0}=1064 \mathrm{~nm}$. A quasi-continuous laser-diode-pumped oscillator was based on a 2.4 at.\%-doped Nd:YAG active crystal (similarly to chapter 4 ) and passively Q-switched by a Cr:YAG saturable absorber. Linearly polarized $5 \mathrm{~ns}, 1 \mathrm{~mJ}$ output pulses were further amplified while using a flashlamp-pumped Nd:YAG amplifier to maximum energy level of $10 \mathrm{~mJ}$. A power supply to $10 \mathrm{~Hz}$ limited the repetition rate.

Figure 6 shows the optical scheme of the parametric Raman laser system and the laser system photo. The active calcite crystal was placed into the external optical cavity consisting of two equal concave mirrors M1 and M2 that were positioned as close as possible to the calcite crystal. The mirrors had a curvature radius of $500 \mathrm{~mm}$ for mode matching with the pump beam being focused by the lens with a focal length of $200 \mathrm{~mm}$. Table 2 presents the cavity mirror reflectivities at the radiation wavelengths of $\lambda_{j}=\left(\lambda_{0}{ }^{-1}-j \cdot v_{R}\right)^{-1}$. Accordingly, the cavity had high quality for SRS oscillation of the only first Stokes component $\left(\lambda_{1}=1203 \mathrm{~nm}\right)$ at single-pass pumping, but the second Stokes SRS component $\left(\lambda_{2}=1384 \mathrm{~nm}\right)$ can be also generated by SRS as a second cascade of conversion in the nonlinear cavity dumping regime [3] under intracavity pumping by the 1203-nm first Stokes SRS component. However, in this configuration, the third Stokes component at $\lambda_{3}=1629 \mathrm{~nm}$ cannot be generated by SRS, because of low quality of the cavity at $\lambda_{2,3}$; therefore, the mechanism of generation at $\lambda_{3}$ can be only parametric.

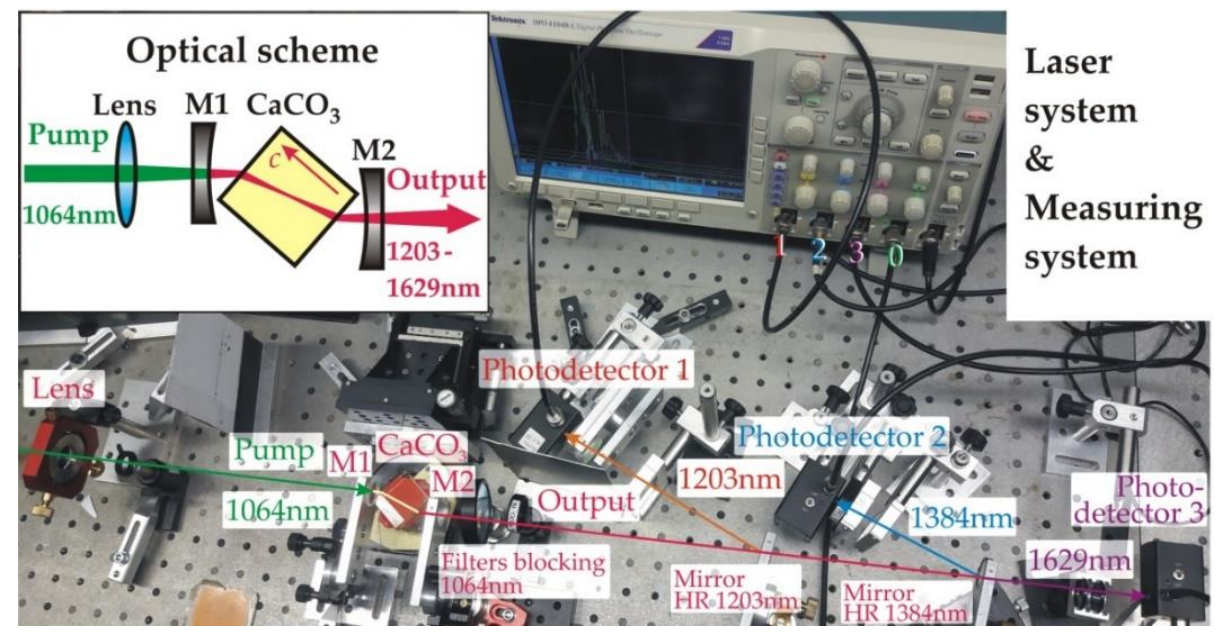

Figure 6. The optical scheme of the extracavity parametric Raman multi-Stokes laser system and the operating laser system photo. 
Table 2. The multi-Stokes laser cavity mirror reflectivities at the radiation wavelengths.

\begin{tabular}{ccccc}
\hline Mirror & $R @ \lambda_{\mathbf{0}}=\mathbf{1 0 6 4} \mathbf{n m}$ & $R @ \lambda_{\mathbf{1}}=\mathbf{1 2 0 3} \mathbf{n m}$ & $R @ \lambda_{\mathbf{2}}=\mathbf{1 3 8 4} \mathbf{n m}$ & $R @ \lambda_{\mathbf{3}}=\mathbf{1 6 2 9} \mathbf{n m}$ \\
\hline M1 \& M2 & $1.2 \%$ & $99.4 \%$ & $20 \%$ & $20 \%$ \\
\hline
\end{tabular}

In order to enhance the 1629-nm third Stokes parametric Raman generation, we tuned the zero-dispersion wavelength of calcite to the value of $\lambda_{d}=1390 \mathrm{~nm}$ by increasing the incidence angle to the calcite crystal up to $49^{\circ}$ (Figure $3 \mathrm{~b}$ ). It allowed for fulfilling the phase matching condition for the second order parametric Raman process, due to $\Delta k_{2}=0$ (see Equation (3), where $j=2$ ) at $\lambda_{2}=1384 \mathrm{~nm}$ (quite a bit less than $\lambda_{d}=1390 \mathrm{~nm}$ ) being the second Stokes SRS component under pumping by our Nd:YAG laser at $\lambda_{0}=1064 \mathrm{~nm}$.

Figure 7 demonstrates the measured spectrum of radiation at the parametric Raman laser output.

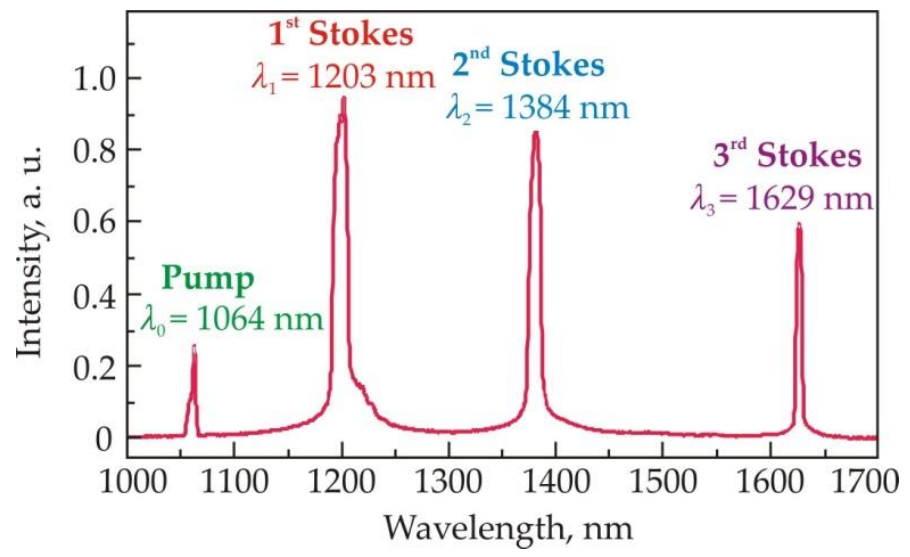

Figure 7. Measured spectrum of radiation at the parametric Raman multi-Stokes laser output.

We can see that three Stokes SRS components were generated that corresponded to the wavelengths of $\lambda_{j}=\left(\lambda_{0}{ }^{-1}-j \cdot v_{R}\right)^{-1}$.

Figure 8a shows the oscillograms of the separated output radiation spectral components registered simultaneously while using four InGaAs PIN photodetectors EOT ET-3500 that were connected to the four-channel oscilloscope Tektronix DPO 4104B-L (Tektronix, Inc., Beaverton, OR, USA) (analog bandwidth $1 \mathrm{GHz}$, temporal resolution $<1 \mathrm{~ns}$ ). The photodiodes measuring the second and third Stokes were connected to the oscilloscope LeCroy-Teledyne SDA 9000 with the highest temporal resolution of $\sim 80$ ps in order to achieve better temporal resolution, see Figure $8 \mathrm{~b}$. It is necessary to note that both of the distances from the laser to the photodetectors and high-speed coaxial cable lengths were equal (see the photo in Figure 6) and, therefore, oscillograms show a real relative temporal position of the radiation components.
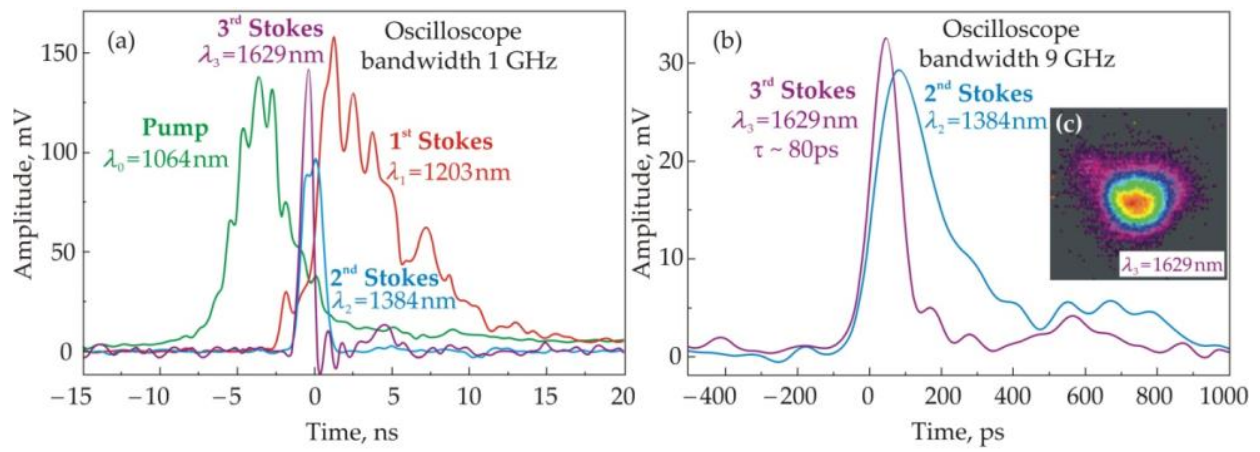

Figure 8. Oscillograms of (a) all the output radiation spectral components and (b) two higher order Stokes components at the highest temporal resolution; and, (c) the measured beam profile of the third Stokes component. 
It can be seen from Figure 8a that a few longitudinal modes were oscillated in the pump laser and in the first Stokes Raman laser, and so the pulses had some modulation. The third Stokes pulse was generated in the temporal region of overlapping pulses of all previous radiation components. It confirms the mechanism of its generation as the zero-dispersion phase-matched second order parametric Raman process, where the first, second, and third Stokes components took part. A distinctive feature of this experimentally observed process from the methodological picture in Figure 1a (in addition to increasing the order of the participating components by one) is the position of the second Stokes pulse at the beginning, not at the end of the first Stokes pulse with the second Stokes pulse shortening down to $\sim 200 \mathrm{ps}$. It can be explained by the nonlinear cavity dumping mechanism of the second Stokes SRS generation [3] with the pulse duration being close to the cavity photon lifetime ( $\sim 100 \mathrm{ps}$ at the cavity mirrors reflectivity of $20 \%)$. Another explanation can be the partial parametric Raman conversion of the first order at not so high wave mismatch of $\Delta k_{1} \approx 9 \mathrm{~cm}^{-1}$ (from Equation (3) at $j=1$ ). Hence, the second Stokes pulse is shortened because of it, but the third Stokes pulse is the shortest (Figure 8b), with the duration of $\sim 80$ ps or even lower. This effect was caused by the phase-matched parametric Raman mechanism (see Figure 1a), i.e., the third Stokes pulse duration was close to the depletion stage of the first Stokes component in the process of its intracavity SRS conversion into the second Stokes component.

Figure $8 \mathrm{c}$ also demonstrates the third Stokes beam profile that is measured by a beam profiling camera Pyrocam III (Ophir-Spiricon, Inc., North Logan, UT, USA). We can see good beam quality, because we made mode matching between the pump spot and the first Stokes Raman laser cavity mode that also resulted in increasing output energy. The output energy of the single 80-ps pulse at $1629 \mathrm{~nm}$ exceeded $30 \mu \mathrm{J}$ for 4-mJ, 5-ns, 1064-nm pumping.

\section{Conclusions}

In conclusion, the conditions of the zero-dispersion phase matching for parametric Raman interactions in birefringent crystals differing by the anisotropy of zero-dispersion wavelength and allowing for the spectral tuning of the zero-dispersion phase-matching condition have been proposed and studied for the method of laser pulse shortening by antiStokes and multi-Stokes parametric Raman conversion. We have demonstrated anti-Stokes $(1168 \mathrm{~nm})$ and multi-Stokes $(1629 \mathrm{~nm})$ picosecond pulse shortening and the self-separation of single 80-ps ultra-short pulse from the zero-dispersion phase-matched parametric Raman lasers that are based on the calcite crystal without using any electro-optical device.

Author Contributions: Conceptualization, S.N.S.; Formal analysis, D.P.T. and S.N.S.; Investigation, M.J., S.N.S. and M.N.E.; Methodology, M.J. and S.N.S.; Resources, M.N.E.; Supervision, S.N.S. and V.K.; Validation, M.J. and V.K.; Visualization, M.J. and S.N.S.; Writing-original draft, M.J. and S.N.S.; Writing-review \& editing, S.N.S., M.J., D.P.T. and V.K. All authors have read and agreed to the published version of the manuscript.

Funding: The research was supported by the ERDF "Center of Advanced Applied Sciences" (No. CZ.02.1.01/0.0/0.0/16_019/0000778) and by the Russian Foundation for Basic Research—Project No 19-02-00723.

Conflicts of Interest: The authors declare no conflict of interest.

\section{References}

1. Boyd, R. Nonlinear Optics, 4th ed.; Academic Press: Cambridge, MA, USA, 2020.

2. Losev, L.L.; Lutsenko, A.P.; Sazonov, S.N. Efficient parametric generation of higher stimulated Raman scattering components with diffraction-limited divergence. Sov. J. Quantum Electron. 1990, 20, 878-879. [CrossRef]

3. Smetanin, S.N.; Jelínek, M., Jr.; Kubecek, V.; Jelínková, H.; Ivleva, L.I.; Shurygin, A.S. Four-wave-mixing and nonlinear cavity dumping of 280 picosecond 2nd Stokes pulse at $1.3 \mu \mathrm{m}$ from Nd:SrMoO 4 self-Raman laser. Laser Phys. Lett. 2015, $13,15801$. [CrossRef]

4. Smetanin, S.N.; Jelínek, M.; Kubeček, V.; Jelínková, H.; Ivleva, L.I. Parametric second Stokes Raman laser output pulse shortening to 300 ps due to depletion of pumping of intracavity Raman conversion. Appl. Phys. A 2016, 122, 260. [CrossRef] 
5. Smetanin, S.N.; Jelínek, M.; Tereshchenko, D.P.; Kubeček, V. Extracavity pumped parametric Raman nanosecond crystalline anti-Stokes laser at $954 \mathrm{~nm}$ with collinear orthogonally polarized beam interaction at tangential phase matching. Opt. Express 2018, 26, 22637-22649. [CrossRef]

6. Smetanin, S.N.; Jelínek, M.; Tereshchenko, D.P.; Shukshin, V.E.; Konyukhov, M.V.; Papashvili, A.G.; Voronina, I.S.; Ivleva, L.I.; Kubeček, V. Nanosecond parametric Raman anti-Stokes $\mathrm{SrWO}_{4}$ laser at $507 \mathrm{~nm}$ with collinear phase matching. Opt. Express 2020, 28, 22919-22932. [CrossRef]

7. Rocco, D.; Vincenti, M.A.; De Angelis, C. Boosting second harmonic radiation from AlGaAsnanoantennas with epsilon-near-zero materials. Appl. Sci. 2018, 8, 2212. [CrossRef]

8. Sautter, J.D.; Xu, L.; Miroshnichenko, A.E.; Lysevych, M.; Volkovskaya, I.; Smirnova, D.A.; Camacho-Morales, R.; Kamali, K.Z.; Karouta, F.; Vora, K.; et al. Tailoring second-harmonic emission from (111)-GaAs Nanoantennas. Nano Lett. 2019, 19, 3905-3911. [CrossRef] [PubMed]

9. Koshelev, K.; Kruk, S.S.; Melik-Gaykazyan, E.V.; Choi, J.-H.; Bogdanov, A.A.; Park, H.-G.; Kivshar, Y. Subwavelength dielectric resonators for nonlinear nanophotonics. Science 2020, 367, 288-292. [CrossRef] [PubMed]

10. Copson, E.T.; Born, M.; Wolf, E. Principles of optics. Math. Gaz. 1961, 45, 274. [CrossRef]

11. Agrawal, G. Nonlinear Fiber Optics, 6th ed.; Academic Press: Cambridge, MA, USA, 2019.

12. Lin, C.; Reed, W.A.; Pearson, A.D.; Shang, H.-T. Phase matching in the minimum-chromatic-dispersion region of single-mode fibers for stimulated four-photon mixing. Opt. Lett. 1981, 6, 493-495. [CrossRef] [PubMed]

13. Basiev, T.T.; Smetanin, S.N.; Shurygin, A.S.; Fedin, A.V. Parametric coupling of frequency components at stimulated Raman scattering in solids. Phys. Uspekhi 2010, 53, 611-617. [CrossRef]

14. Wang, C.; Lv, D. Numerical modeling of the intracavity coherent anti-Stokes Raman laser. J. Opt. Soc. Am. B 2020, 37, 3184-3193. [CrossRef]

15. Smetanin, S.N.; Doroshenko, M.E.; Ivleva, L.I.; Jelínek, M.; Kubeček, V.; Jelinková, H. Low-threshold parametric Raman generation of high-order Raman components in crystals. Appl. Phys. A 2014, 117, 225-234. [CrossRef]

16. Chiao, R.; Stoicheff, B.P. Angular dependence of maser-stimulated Raman radiation in calcite. Phys. Rev. Lett. 1964, 12, 290-293. [CrossRef]

17. Giordmaine, J.A.; Kaiser, W. Light scattering by coherently driven lattice vibrations. Phys. Rev. 2002, 144, 676-688. [CrossRef]

18. Bass, M.; DeCusatis, C.; Enoch, J.; Lakshminarayanan, V.; Li, G.; MacDonald, C.; Mahajan, V.; van Stryland, E. Handbook of Optics. Volume IV: Optical Properties of Materials, Nonlinear Optics, Quantum Optics, 3rd ed.; The McGraw-Hill Companies Inc.: New York, NY, USA, 2010.

19. Shen, Y.R. Principles of Nonlinear Optics; John Wiley \& Sons Inc.: New Jersey, NJ, USA, 2003.

20. Kaminskii, A.A.; Ueda, K.I.; Eichler, H.J.; Kuwano, Y.; Kouta, H.; Bagaev, S.N.; Chyba, T.H.; Barnes, J.C.; Gad, G.M.A.; Murai, T.; et al. Tetragonal vanadates $\mathrm{YVO}_{4}$ and $\mathrm{GdVO}_{4}-\mathrm{New}$ efficient $\chi^{(3)}$ materials for Raman lasers. Opt. Commun. 2001, 194, 201-206. [CrossRef]

21. Zagumennyi, A.I.; Mikhailov, V.A.; Shcherbakov, I.A. Rare Earth Ion Lasers—Nd3+. Handbook of Laser Technology and Applications, Volume II: Laser Design and Laser Systems; Institute of Physics Publishing: Bristol, UK, 2004.

22. Jelínek, M.; Smetanin, S.; Páta, P.; Fliegel, K.; Kubecek, V. Generation of 120 ps, 1168 nm anti-Stokes pulses from the all-solid-state, self-mode-locked, parametric Raman $\mathrm{CaCO}_{3}$ laser with intracavity pumping by $1338 \mathrm{~nm}$ Nd:YAG laser. Photonics Devices Syst. VII 2017, 10603, 50. [CrossRef] 